\title{
Role of video capsule endoscopy in patients with constitutional mismatch repair deficiency (CMMRD) syndrome: report from the International CMMRD Consortium
}

\section{(๑) $\circledast \ominus$}

\author{
Authors \\ Y. Shimamura ${ }^{1}$, C. M. Walsh², S. Cohen ${ }^{3}$, M. Aronson ${ }^{4}$, U. Tabori ${ }^{5}$, P. P. Kortan ${ }^{1}$, C. A. Durno ${ }^{4,6}$, and the International \\ BMMRD Consortium
}

Institutions

1 Division of Gastroenterology, St. Michael's Hospital, University of Toronto, Toronto, Canada

2 Division of Gastroenterology, Hepatology and Nutrition, the Research and Learning Institutes, Hospital for Sick Children, Department of Paediatrics and the Wilson Centre, University of Toronto, Toronto, Canada

3 Pediatric Gastroenterology Unit of “Dana-Dwek” Children's Hospital, Tel Aviv Sourasky Medical Center, affiliated to Sackler Faculty of Medicine, Tel Aviv University, Tel Aviv, Israel

4 Zane Cohen Centre for Digestive Diseases, Mount Sinai Hospital, Toronto, Canada

5 Division of Haematology and Oncology, Hepatology and Nutrition, Hospital for Sick Children, University of Toronto, Toronto, Canada

6 Division of Gastroenterology, Hepatology and Nutrition, Hospital for Sick Children, University of Toronto, Toronto, Canada

submitted 26.9.2017

accepted after revision 20.2.2018

\section{Bibliography}

DOI https://doi.org/10.1055/a-0591-9054 |

Endoscopy International Open 2018; 06: E1037-E1043

(c) Georg Thieme Verlag KG Stuttgart · New York ISSN 2364-3722

\section{Corresponding author}

Carol Durno, MD, MSc, FRCPC, Hospital for Sick Children, Division of Gastroenterology, Hepatology and Nutrition, 555 University Ave, Toronto, ON, Canada M5G 1X8

Fax: + 4168136531

carol.durno@sickkids.ca

\section{ABSTRACT}

Background and study aims Constitutional mismatch repair deficiency (CMMRD) syndrome, also known as biallelic mismatch repair deficiency (BMMRD) syndrome is a rare autosomal-recessive genetic disorder that has a high mortality due to malignancy in childhood and early adulthood. The small bowel phenotype in CMMRD is not well described and surveillance protocols for small bowel cancer have not been well established. This study was conducted to evaluate the usefulness and clinical impact of video capsule endoscopy (VCE) for small bowel surveillance.

Patients and methods We retrospectively reviewed the prospectively maintained International CMMRD Consortium database. Treating physicians were contacted and VCE report data were extracted using a standardized template.

Results Among 58 patients included in the database, 38 VCE reports were collected from 17 patients. Polypoid lesions were first detected on VCE at a median age of 14 years (range: $4-17)$. Of these, $39 \%$ in 7 patients (15/38) showed large polypoid lesions $(>10 \mathrm{~mm}$ ) or multiple polyps that prompted further investigations. Consequently, three patients were diagnosed with small bowel neoplasia including one patient with adenocarcinoma. Small bowel neoplasia and/or cancer were confirmed histologically in $35 \%$ of the patients (6/17) who had capsule surveillance and the lesions in half of these patients were initially visualized on VCE. Multiple polyps were identified on eight VCEs that were completed on three patients. Ten VCEs (28\%) were incomplete due to slow bowel transit; none required capsule removal.

Conclusions Small bowel surveillance in patients with CMMRD should be initiated early in life. VCE has the potential to detect polyps; however, small bowel neoplasias are often proximal and can be missed, emphasizing the importance of concurrent surveillance with other modalities.

Meeting presentations Digestive Disease Week 2017 and World Congress of Pediatric Gastroenterology, Hepatology and Nutrition 2016. 


\section{Introduction}

Small bowel cancer is a rare disease accounting for $3 \%$ of all gastrointestinal malignancies [1]. Incidence of small bowel cancer is increased in polyposis syndromes such as Lynch syndrome, familial adenomatous polyposis (FAP), and Peutz-Jeghers Syndrome (PJS) [2-5]. Lynch syndrome is a well described autosomal-dominant cancer predisposition syndrome characterized by increased risk of colorectal cancer and extracolonic malignancies including small bowel, endometrial and genitourinary cancers [6]. Lynch syndrome is associated with heterozygous (monoallelic) germline mutations that affect DNA mismatch repair (MMR) genes, mainly $\mathrm{MLH1}, \mathrm{MSH} 2$, MSH6, and PMS2. A rare and more virulent cancer predisposition syndrome results when biallelic MMR gene mutations are inherited, termed biallelic constitutional MMR deficiency (CMMRD). This disorder, which is also called biallelic MMR deficiency (BMMRD), is a rare autosomal-recessive genetic disorder that has a high mortality due to malignancy in childhood and early adulthood [7]. CMMRD is characterized by a broad spectrum of early-onset tumors including gastrointestinal cancers and brain and hematological malignancies and is frequently accompanied by café-au-lait macules [7-11]. Synchronous and metachronous gastrointestinal and/or extraintestinal cancers occur frequently in CMMRD [12]. The association between colorectal cancer and small bowel adenocarcinoma also has been reported $[8,12,13]$. Based on small case series and case reports, prevalence of small bowel cancer in CMMRD is $10 \%$ to $16 \%$ and the median age at diagnosis of small bowel cancer is 28 years, with a range of 11 to 42 years [14].

The recent American Gastroenterology Association's (AGA) clinical practice guideline for hereditary polyposis syndromes recommends video capsule endoscopy (VCE) as a part of ongoing surveillance to enable visualization of the entire small bowel noninvasively [15]. Although VCE is a useful surveillance modality for detecting small bowel polyps in patients with FAP, PJS, and Lynch syndrome [16-20], the utility of VCE surveillance in CMMRD patients remains unstudied. The Familial Gastrointestinal Cancer Registry (FGICR) at Mount Sinai Hospital and the Hospital for Sick Children (Toronto, Canada) joined to establish an International BMMRD Consortium in 2010 to better define the clinical and genetic characteristics of CMMRD. This International CMMRD Consortium, and the European Consortium for the care of CMMRD subjects currently recommend starting small bowel cancer surveillance with VCE at 8 and 10 years of age, respectively $[12,14,21]$. A consensus statement by the US Multi-Society Task Force on colorectal cancer recommends annual surveillance by upper endoscopy and VCE beginning at age 8 years [14]. However, the validity of these recommendations has not been examined. This is the first study to evaluate the usefulness and clinical impact of VCE for small bowel neoplasia and cancer surveillance in CMMRD patients. We have previously reported the gastrointestinal phenotype, including small bowel neoplasia, from the International CMMRD Consortium [12]. This study was conducted to further evaluate the feasibility of VCE surveillance in CMMRD patients.

\section{Patients and methods}

We retrospectively reviewed the prospectively maintained International BMMRD Consortium database between January 2010 and May 2016, during which time there were no established VCE guidelines for this patient population. Patients from 11 countries who were proven carriers of two MMR gene mutations were included in this dataset. Treating physicians were contacted and VCE report data were extracted using a standardized template. Data extracted included age, sex, MMR gene mutations, procedure date, location and number of polyps detected by VCE and resultant investigations. Treating physicians were asked if VCE findings led to further investigation such as surgery, small bowel enteroscopy, or any other interventions. A polyp was defined as a discrete tissue protruding into the small bowel lumen. Lesion location was estimated by analyzing VCE transit time between the first image of the duodenum and the ileocecal valve or ileocolonic anastomosis. In cases with an incomplete VCE study, we considered the last image of the small bowel as the terminal ileum in order to estimate the location of the lesion utilizing data on VCE transit time. The study was approved by Mount Sinai Hospital, Hospital for Sick Children and participating institutions' ethics review committees and informed consent was obtained from all patients.

\section{Results}

\section{Summary of VCE results}

Fifty-eight patients (37 kindreds) were included in the International BMMRD Consortium database. Thirty-eight VCE reports were collected from 17 patients ( $\triangleright$ Table 1 ). Most of the other patients had no small bowel surveillance, except two patients who underwent magnetic resonance enterography (MRE). The initial VCE was performed at a median age of 14 years (range: 4-31). Four video capsules were delivered endoscopically. Polypoid lesions were detected in $63 \%$ of VCEs (24/38) conducted on nine patients. These polypoid lesions included lesions suspected to be adenomatous polyps, hyperplastic polyps, malignant-appearing lesions, and enlarged lymphoid nodular hyperplasia (LNH). All polypoid lesions had morphologic features of sessile or flat elevated lesions (Paris endoscopic classification 0-Is and 0-Ila [22]) ( Fig.1). Polypoid lesions were first detected on VCE at a median age of 14 years (range: 4 -17). Thirty-nine percent of VCEs 15/38) in 7 patients showed large polypoid lesions $(>10 \mathrm{~mm})$ or multiple polyps which prompted further investigations, including five laparotomies and two double-balloon enteroscopies ( $\$$ Table 2 ). Consequently, 3 of the 17 patients in our cohort were diagnosed with small bowel neoplasia on histology, including an adenocarcinoma in Patient \#14 and tubular adenomas with high-grade dysplasia in Patients \#2 and \#15. The other polypoid lesions detected on VCE were either not assessed by endoscopy or were diagnosed as hyperplastic polyps or LNH. Decisions regarding further evaluation and polyp excision were made at the discretion of the clinicians looking after each patient. VCE did not aid in diagnosis of lesions in three additional patients in the cohort who were diagnosed with either histologically confirmed small 


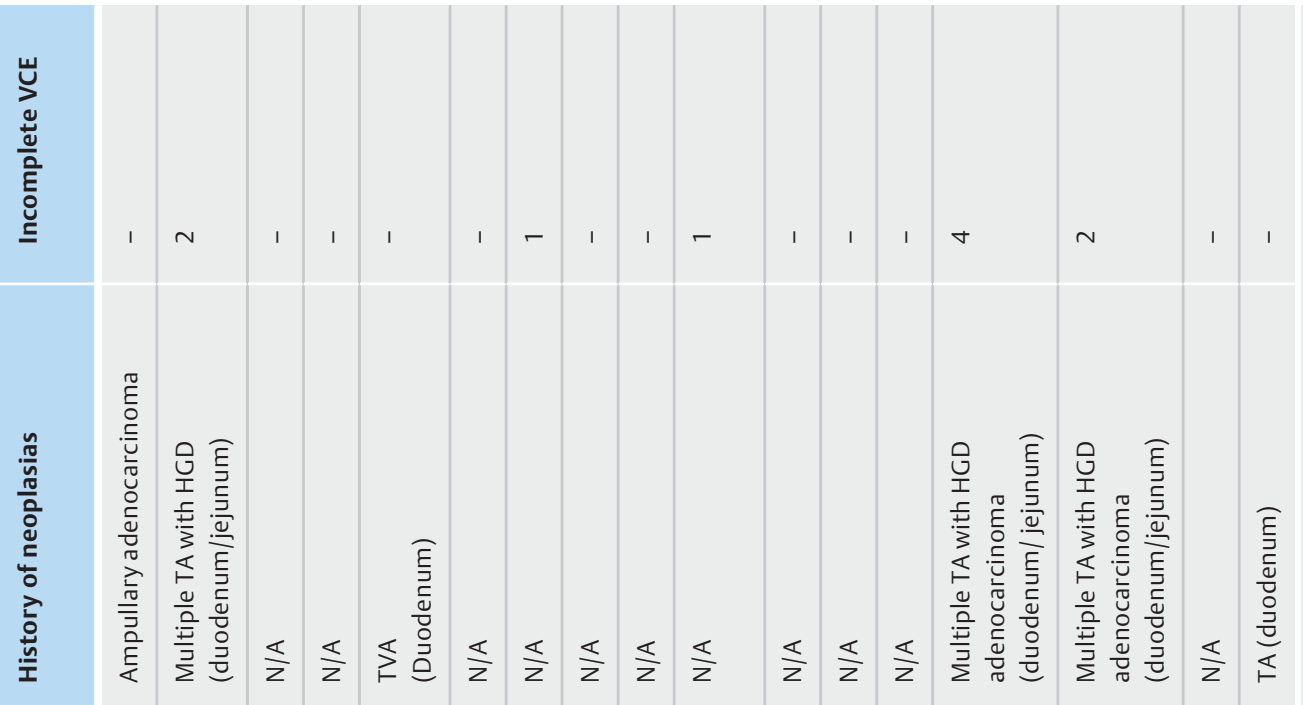

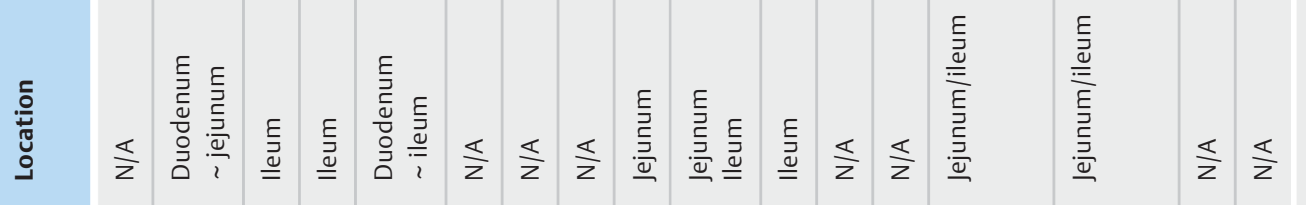

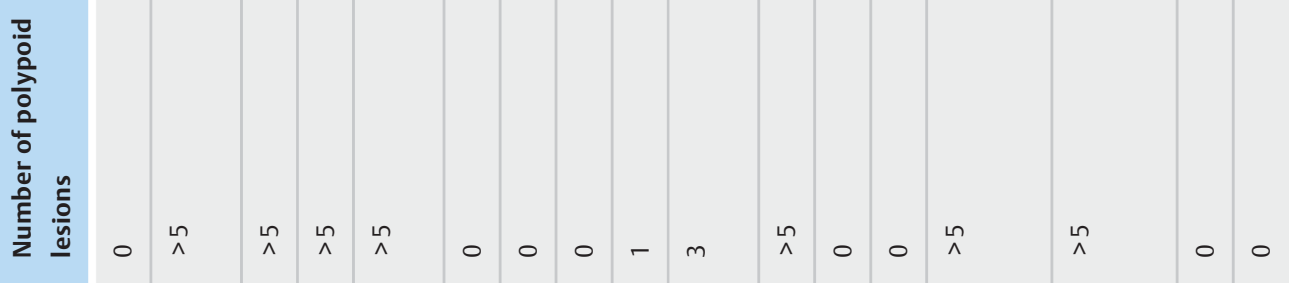

\begin{tabular}{|c|c|c|c|c|c|c|c|c|c|c|c|c|c|c|c|c|c|}
\hline 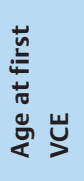 & $\simeq$ & $\stackrel{m}{r}$ & $\stackrel{m}{=}$ & $\mp$ & $\stackrel{\nabla}{\square}$ & $\stackrel{\nabla}{\ulcorner}$ & $\bar{m}$ & $\stackrel{\nabla}{\sim}$ & $\nabla$ & $\simeq$ & $\stackrel{\Xi}{\rightleftharpoons}$ & $\stackrel{\bullet}{-}$ & $\stackrel{\circ}{\square}$ & $\stackrel{\nabla}{\square}$ & $\stackrel{\bullet}{\leftarrow}$ & $\stackrel{\llcorner}{\sim}$ & $\approx$ \\
\hline 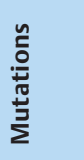 & $\begin{array}{l}\frac{0}{1} \\
\stackrel{5}{\Sigma} \\
\Sigma\end{array}$ & $\sum_{a}^{N}$ & $\begin{array}{l}\frac{0}{1} \\
\frac{1}{N}\end{array}$ & $\begin{array}{l}\frac{0}{1} \\
\stackrel{5}{\Sigma}\end{array}$ & 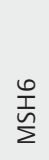 & $\sum_{a}^{\tilde{N}}$ & $\sum_{a}^{N}$ & $\sum_{a}^{N}$ & 司 & $\begin{array}{l}\frac{0}{1} \\
\stackrel{5}{\Sigma}\end{array}$ & $\sum_{0}^{N}$ & $\begin{array}{l}\frac{0}{1} \\
\frac{1}{\Sigma}\end{array}$ & $\sum_{0}^{N}$ & $\overline{\underline{I}}$ & $\stackrel{\bar{I}}{\Sigma}$ & $\frac{0}{\frac{0}{\omega}}$ & $\begin{array}{l}\frac{0}{1} \\
\frac{5}{\Sigma} \\
\Sigma\end{array}$ \\
\hline 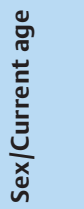 & $\frac{\vec{m}}{\underline{n}}$ & 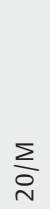 & $\sum_{I}$ & $\stackrel{\sum}{\underline{n}}$ & $\stackrel{\stackrel{\omega}{\infty}}{\sim}$ & $\sum_{0}$ & $\underset{m}{\stackrel{u}{N}}$ & 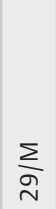 & $\begin{array}{l}\tilde{\Xi} \\
\tilde{\Xi} \\
\stackrel{\Xi}{~} \\
\Delta\end{array}$ & $\begin{array}{l}\bar{\Xi} \\
\widetilde{\Xi} \\
\tilde{\Xi} \\
\tilde{\Xi}\end{array}$ & $\underset{\Sigma}{\Sigma}$ & $\sum_{\infty}$ & $\underset{I}{\Sigma}$ & $\stackrel{\stackrel{u}{\bar{v}}}{\bar{N}}$ & $\frac{\omega}{N}$ & $\frac{u}{m}$ & $\frac{\frac{\omega}{\infty}}{\sim}$ \\
\hline & - & $\sim$ & $m$ & $\nabla$ & in & 0 & $r$ & $\infty$ & $a$ & $\stackrel{\circ}{\circ}$ & $=$ & $\simeq$ & $\stackrel{m}{\square}$ & $\stackrel{\Xi}{\check{L}}$ & $\stackrel{\sim}{\sim}$ & $\stackrel{\bullet}{\circ}$ & $\approx$ \\
\hline
\end{tabular}



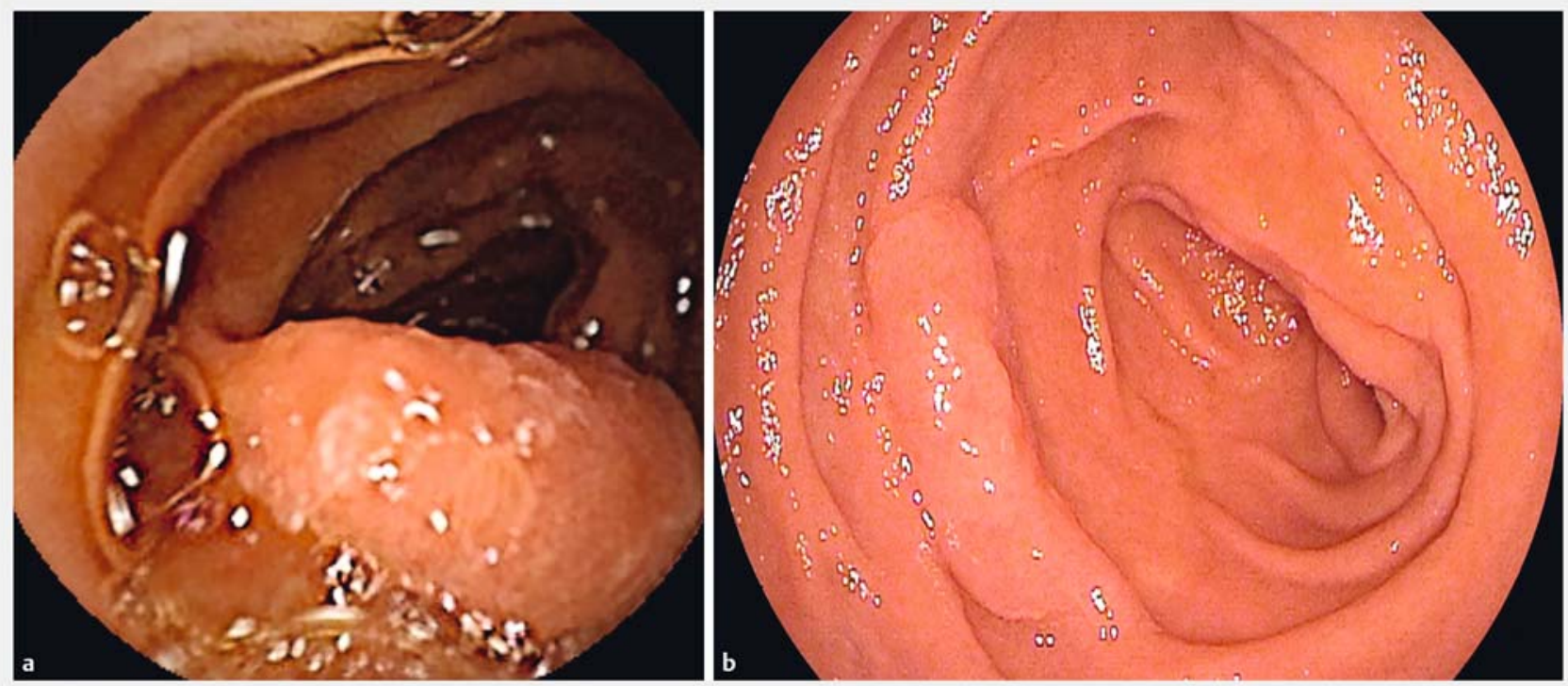

Fig. 1 VCE image of a sessile polyp in the jejunum. Further investigation was done with antegrade double-balloon enteroscopy.

- Table 2 Results of further investigations of possible suspicious lesions identified on VCE.

\begin{tabular}{|c|c|c|c|c|c|}
\hline Age & $\begin{array}{l}\text { Capsule } \\
\text { number }\end{array}$ & VCE finding & Location & Procedure & Final diagnosis \\
\hline 14 & 1 & 4 polyps $(10 \mathrm{~mm})$ & Proximal jejunum & $\begin{array}{l}\text { Laparotomy } \\
\text { Small bowel resection }\end{array}$ & TA with HGD \\
\hline 15 & 2 & $\begin{array}{l}10 \text { polyps }(<5 \mathrm{~mm}) \\
2 \text { polyps }(10 \mathrm{~mm})\end{array}$ & $\begin{array}{l}\text { Proximal jejunum } \\
\text { Distal jejunum }\end{array}$ & Laparotomy-assisted enteroscopy & $\begin{array}{l}\text { TA with HGD } \\
\text { (Jejunal polyp) }\end{array}$ \\
\hline 16 & 3 & $\begin{array}{l}>5 \text { Polyps }(<5 \mathrm{~mm}) \\
1 \text { polyp }(10 \mathrm{~mm})\end{array}$ & $\begin{array}{l}\text { Proximal jejunum } \\
\text { Proximal jejunum }\end{array}$ & Laparotomy-assisted enteroscopy & $\begin{array}{l}\text { TA } \\
\text { (Duodenal and jejunal } \\
\text { polyp) }\end{array}$ \\
\hline 15 & 1 & $>5$ polyps $(<5 \mathrm{~mm})$ & Ileum & Early colonoscopy & No abnormalities \\
\hline 16 & 1 & $>5$ polyps $(<5 \mathrm{~mm})$ & Ileum & Early colonoscopy & No abnormalities \\
\hline 13 & 1 & $>5$ polyps $(<5 \mathrm{~mm})$ & Ileum & Early colonoscopy & No abnormalities \\
\hline 15 & 1 & $>5$ polyps $(<5 \mathrm{~mm})$ & Ileum & Early colonoscopy & Lymphoid hyperplasia \\
\hline 16 & 2 & 1 Polyp (<5 mm) & Jejunum & Antegrade DBE & No abnormalities \\
\hline 15 & 1 & $>5$ polyps $(<5 \mathrm{~mm})$ & Ileum & Early colonoscopy & No abnormalities \\
\hline 14 & 1 & $<5$ polyps $(<5 \mathrm{~mm})$ & Jejunum & Push enteroscopy & TA with LED \\
\hline 16 & 2 & 5 polyps & Jejunum & Laparotomy & Jejunal adenocarcinoma \\
\hline 17 & 3 & 5 polyps & Jejunum & $\begin{array}{l}\text { Laparotomy-assisted enteroscopy } \\
\text { and proximal small bowel resection }\end{array}$ & TA \\
\hline 20 & 4 & LST $20 \mathrm{~mm}$ & Proximal jejunum & Antegrade DBE & TA with HGD \\
\hline 17 & 1 & $\begin{array}{l}2 \text { polyps } \\
\text { Mucosal irregularities }\end{array}$ & Proximal Jejunum & Push enteroscopy & TA with HGD \\
\hline 22 & 2 & $\begin{array}{l}<5 \text { polyps } \\
\text { Mucosal irregularities }\end{array}$ & Jejunum & Push enteroscopy & TA with LGD \\
\hline
\end{tabular}


bowel neoplasia (Patient \#5 and \#17) and/or cancer (Patients \#1) during this study period. The aforementioned Patient \#15 was also diagnosed with a small bowel cancer for which VCE did not aid in diagnosis.

\section{Small bowel cancer}

In total, three patients in this cohort were diagnosed with small bowel cancer (SBC) during this study period, one of which was with the aid of VCE (Patient \#14). The first patient was a 12year-old female (Patient \#1) with a history of colon cancer who was diagnosed with ampullary adenocarcinoma on screening gastroscopy. She underwent VCE to exclude other small bowel lesions prior to Whipple procedure. The VCE study failed to detect this known ampullary cancer. She underwent surgery and currently is well without recurrence for 2 years. In addition, there were two siblings who were diagnosed with SBC. The younger sibling was a 15-year-old female (Patient \#14) who had a history of transduodenal resection of an ampullary adenoma at age 11 . She was found to have multiple polyps in the jejunum on VCE, findings which prompted laparotomy to remove duodenal and jejunal polyps. One of her polyps revealed a jejunal adenocarcinoma with lymph node metastases that was subsequently treated with chemotherapy. A VCE conducted 12 months prior had revealed multiple duodenal sessile lesions and duodenal adenoma; however, no jejunal lesion was visualized. An MRI performed 5 months prior to her initial VCE also did not reveal any polyps.

This patient subsequently developed a small bowel polyp in the proximal jejunum which was detected with VCE and endoscopic resection with double-balloon enteroscopy revealed a tubular adenoma with high-grade dysplasia. This lesion was in the distal jejunum, requiring double-balloon enteroscopy as it was not reachable with gastroscopy and/or push enteroscopy. The patient is currently being followed by push enteroscopy twice per year and VCE annually. No recurrence of small bowel cancer and no lesion larger than $10 \mathrm{~mm}$ has been identified within the follow-up period of 78 months. The older sibling was a 20-year-old female (Patient \#15) under small bowel cancer surveillance who was diagnosed with duodenal and jejunal adenocarcinoma 40 months after a normal baseline VCE. Surveillance had included a normal esophagogastroduodenoscopy completed 16 months prior to diagnosis and two normal MRE completed 26 and 31 months prior to diagnosis. A subsequent MRE identified a short segment of irregular mucosal thickening of the proximal jejunum $2.2 \mathrm{~cm}$ in length just beyond the ligament of Treitz. This prompted a push enteroscopy which confirmed duodenal and jejunal adenocarcinoma that were surgically resected. The patient is currently undergoing surveillance postoperatively with push enteroscopy twice per year and VCE annually. No recurrence of duodenal or jejunal cancer and no lesion larger than $10 \mathrm{~mm}$ has been identified within the follow-up period of 48 months.

\section{Multiple small bowel polyps}

Eight VCEs carried out on three patients, including the aforementioned two siblings (Patients \#2, \#14, and \#15), showed multiple polyps in the small bowel. This was distinct from other patients who had few $(1-2)$ or no polyps. These findings prompted multiple push enteroscopies, double-balloon enteroscopies and several intraoperative enteroscopies. Patient \#2 is currently undergoing surveillance with push enteroscopy every 3 to 4 months, and annual MRE. Small bowel adenomas with low-grade dysplasias have been identified on multiple surveillance push enteroscopies and were resected with polypectomy. This patient has not developed small bowel cancer in the 9 years since his initial endoscopic surveillance.

\section{Missed lesions on VCE}

In addition to the aforementioned Patients \#1 and \#15, there were two additional patients (Patients \#5 and \#17) in this cohort who had negative VCEs who were subsequently found to have an adenomatous lesion on endoscopy. Patient \#5 was found to have a tubulovillous adenoma in the duodenum on gastroscopy performed 16 months after initial VCE. Patient \#17 had a tubular adenoma in the duodenum that was detected via side-viewing esophagogastroduodenoscopy performed 4 months after a negative VCE. Moreover, this patient had another negative VCE 2 years later and was subsequently found to have a second tubular adenoma in the duodenum that was detected via side-viewing esophagogastroduodenoscopy performed 1 month after the negative VCE. These were considered lesions missed by VCE or false-negative VCE studies. Of note, these lesions were within reach of a conventional gastroduodenoscope.

\section{Incomplete VCE}

Ten VCEs (28\%) performed on five patients failed to examine the entire small intestine as the bowel transit time exceeded the capsule battery life. Four of these patients had undergone colectomy with ileorectal anastomosis and three of the five patients had multiple surgeries (two had small bowel resections and one underwent partial gastrectomy). These previous surgeries may have caused prolonged small bowel transit time. However, all capsules passed spontaneously, and none required a capsule removal procedure.

\section{Discussion}

Upper endoscopy and VCE are the diagnostic modalities currently recommended by the US Multi-Society Task Force for evaluating the small bowel in CMMRD patients; however, the clinical impact of VCE surveillance is unknown. Several important findings have emerged from our study in this regard. First, this study highlights the need for small bowel surveillance in CMMRD patients commencing early in childhood. Within our cohort, $18 \%$ of the patients (3/17) were diagnosed with small bowel neoplasia confirmed histologically during this study period. This is much higher than the $1.5 \%$ prevalence of small bowel neoplasia reported in asymptomatic patients with Lynch Syndrome [23]. Although VCE seems to be a useful diagnostic modality, it is too early to conclude that VCE should be the standard of care in surveillance programs for CMMRD patients. While all small bowel cancers identified by VCE were diagnosed at an early stage and amenable to resection, use of VCE for 
small bowel surveillance is still controversial due to the possibility of a false-negative study [24-26]. In our cohort, there were four patients (\#1, \#5, \#15, and \#17) who had negative VCEs who were subsequently found to have neoplasias in the duodenum. Similar findings have been reported in previous studies which have shown that endoscopy is superior to VCE for detection of duodenal polyps $[18,25,27]$. As compared with endoscopy, VCE may have a decreased sensitivity for detection of duodenal lesion due to the rapid transit time of the capsule through the duodenum. Interestingly, all small bowel cancers seen in this cohort were in the duodenum and the proximal jejunum. Therefore, aside from VCE surveillance, we should emphasize the importance of performing push enteroscopy and/ or side-viewing duodenoscopy.

A second important study finding is that three patients were noted to have multiple polyps visualized on 10 of 12 VCEs. Based on these findings, we can speculate that there may be a subset of patients who are predisposed to small bowel polyps and small bowel cancer within the CMMRD population. However, given our small sample size, no genotype phenotype correlation with small bowel polyposis could be made.

Finally, our study results indicate that there may be a high risk of capsule retention. Twenty-eight percent of VCEs (performed on five patients) yielded incomplete small bowel observation as the bowel transit time exceeded the capsule battery life. Four of these patients had previous surgery which may have caused prolonged transit time. Although there was no capsule retention necessitating a capsule retrieval procedure, it is important to inform patients and their family of the risks of capsule retention and bowel obstruction. Use of patency capsules in patients with known and/or suspected small bowel strictures and potential capsule delivery beyond proximal strictures may be useful strategies to minimize risk of capsule retention. Longer VCE battery life may improve completion rates; however, this may not affect the rate of positive findings as all small bowel cancers in this cohort were in the duodenum and the proximal jejunum [28].

In summary, although VCE is a useful modality for small bowel surveillance, we should not solely depend on VCE findings, especially given its limitations, which include the potential for incomplete studies, a high rate of false-positive exams and the possibility of a false-negative study. Additional endoscopic modalities should be considered to complement VCE. We recommend push enteroscopy with careful inspection of the ampullary region. For pediatric patients, push maneuver with a gastroscope may be an alternative modality to observe the distal duodenum and proximal jejunum. Although we cannot draw conclusions regarding the ideal modality for small bowel surveillance, initiation of small bowel surveillance earlier than age 10 should be considered, in keeping with current recommendations from the International CMMRD Consortium, the European Consortium for the care of CMMRD subjects and the US MultiSociety Task Force on colorectal cancer.

This study has several limitations. First, the sample size is too small to draw conclusions regarding the diagnostic yield of capsule endoscopy in CMMRD patients. A larger prospective study of small bowel screening with VCE is required to better under- stand the small bowel phenotype of this population. Although there were cases that prompted further investigation, most of the polypoid lesions seen on VCE were not confirmed with panenteroscopy, considering the invasive nature of this procedure. Only highly suspicious lesions, such as large or multiple lesions, underwent further investigation. Third, we were unable to compare small bowel surveillance modalities due to lack of data on MRE and other radiological examinations in this study population. In previous studies comparing small bowel evaluation modalities, VCE has demonstrated higher sensitivity for polyps than radiological surveillance, including small bowel follow through and MRE. However, it is still debatable which modality has the highest overall diagnostic accuracy and specificity. Lastly, although we were able to collect VCE reports for analysis, we could not review all VCE images. There may be cases in which polyps were missed by the physicians who interpreted the VCE and/or cases for which physicians did not fully report their findings.

\section{Conclusions}

VCE has the potential to detect polyps in the CMMRD population and surveillance of patients for neoplastic lesions may be feasible; however, small bowel neoplasias are often proximal and may be missed on VCE, emphasizing the importance of concurrent surveillance with other endoscopy such as push enteroscopy with careful inspection in the ampullary region. Looking to the future, it will be important to assess the accuracy of concurrent evaluation using other modalities and, thereby, to build an evidenced-based small bowel surveillance protocol for patients with CMMRD syndrome. The clinical usefulness of small bowel screening in CMMRD patients should be confirmed with large prospective studies.

\section{Competing interests}

None

References

[1] Raghav K, Overman MJ. Small bowel adenocarcinomas - existing evidence and evolving paradigms. Nature Rev Clin Oncol 2013; 10: 534 544

[2] Schulmann K, Brasch FE, Kunstmann E et al. HNPCC-associated small bowel cancer: clinical and molecular characteristics. Gastroenterology 2005; 128: $590-599$

[3] Offerhaus G], Giardiello FM, Krush A] et al. The risk of upper gastrointestinal cancer in familial adenomatous polyposis. Gastroenterology 1992; 102: 1980- 1982

[4] Koornstra J]. Small bowel endoscopy in familial adenomatous polyposis and Lynch syndrome. Best Pract Res Clin Gastroenterol 2012; 26: $359-368$

[5] Giardiello FM, Brensinger JD, Tersmette AC et al. Very high risk of cancer in familial Peutz-Jeghers syndrome. Gastroenterology 2000; 119: $1447-1453$ 
[6] Hata K, Yamamoto Y, Kiyomatsu T et al. Hereditary gastrointestinal cancer. Surgery Today 2016; 46: 1115-1122

[7] Durno CA, Sherman PM, Aronson M et al. Phenotypic and genotypic characterisation of biallelic mismatch repair deficiency (BMMR-D) syndrome. European journal of cancer (Oxford, England: 1990) 2015; 51: $977-983$

[8] Lavoine N, Colas C, Muleris M et al. Constitutional mismatch repair deficiency syndrome: clinical description in a French cohort. J Med Genetics 2015; 52: 770 - 778

[9] Elhasid R, Dvir R, Rosenfeld Keidar $\mathrm{H}$ et al. Management of acute myeloblastic leukemia in a child with biallelic mismatch repair deficiency. J Ped Hem Onc 2015; 37: e490-493

[10] Amayiri N, Tabori U, Campbell B et al. High frequency of mismatch repair deficiency among pediatric high grade gliomas in Jordan. Int J Cancer 2016; 138: 380 - 385

[11] Wimmer K, Kratz CP, Vasen HF et al. Diagnostic criteria for constitutional mismatch repair deficiency syndrome: suggestions of the European consortium 'care for CMMRD' (C4CMMRD). J Med Genetics 2014; 51: 355-365

[12] Aronson M, Gallinger S, Cohen Z et al. Gastrointestinal findings in the largest series of patients with hereditary biallelic mismatch repair deficiency syndrome: report from the International Consortium. Am J Gastroenterol 2016; 111: 275-284

[13] Durno CA, Holter S, Sherman PM et al. The gastrointestinal phenotype of germline biallelic mismatch repair gene mutations. Am J Gastroenterol 2010; 105: 2449-2456

[14] Durno C, Boland CR, Cohen S et al. Recommendations on surveillance and management of biallelic mismatch repair deficiency (BMMRD) syndrome: A consensus statement by the US Multi-Society Task Force on Colorectal Cancer. Gastroenterology 2017; 152: 1605-1614

[15] Enns RA, Hookey L, Armstrong D et al. Clinical practice guidelines for the use of video capsule endoscopy. Gastroenterology 2017; 152: $497-514$

[16] Schulmann K, Hollerbach S, Kraus K et al. Feasibility and diagnostic utility of video capsule endoscopy for the detection of small bowel polyps in patients with hereditary polyposis syndromes. Am J Gastroenterol 2005; 100: $27-37$
[17] laquinto G, Fornasarig M, Quaia M et al. Capsule endoscopy is useful and safe for small-bowel surveillance in familial adenomatous polyposis. Gastrointest Endosc 2008; 67: 61-67

[18] Tescher P, Macrae FA, Speer T et al. Surveillance of FAP: a prospective blinded comparison of capsule endoscopy and other $\mathrm{Gl}$ imaging to detect small bowel polyps. Hered Cancer Clin Pract 2010; 8: 3

[19] Baichi MM, Arifuddin RM, Mantry PS. Metachronous small bowel adenocarcinomas detected by capsule endoscopy in a patient with hereditary nonpolyposis colorectal cancer. Dig Dis Sci 2007; 52: 1134 1136

[20] Marmo R, Rotondano G, Riccio G et al. Small-bowel adenocarcinoma diagnosed via capsule endoscopy in a patient found to have hereditary nonpolyposis colorectal cancer. Gastrointest Endosc 2007; 65: $524-525$, discussion 525

[21] Vasen HF, Ghorbanoghli Z, Bourdeaut F et al. Guidelines for surveillance of individuals with constitutional mismatch repair-deficiency proposed by the European Consortium "Care for CMMR-D" (C4CMMR-D). J Med Genet 2014; 51: 283-293

[22] Anonymous. Update on the paris classification of superficial neoplastic lesions in the digestive tract. Endoscopy 2005; 37: 570-578

[23] Haanstra JF, Al-Toma A, Dekker E et al. Prevalence of small-bowel neoplasia in Lynch assessed by video capsule endoscopy. Gut 2015; 64: $1578-1583$

[24] Ross A, Mehdizadeh S, Tokar J et al. Double balloon enteroscopy detects small bowel mass lesions missed by capsule endoscopy. Dig Dis Sci 2008; 53: $2140-2143$

[25] Baichi MM, Arifuddin RM, Mantry PS. Small-bowel masses found and missed on capsule endoscopy for obscure bleeding. Scand J Gastroenterol 2007; 42: $1127-1132$

[26] Chong AK, Chin BW, Meredith CG. Clinically significant small-bowel pathology identified by double-balloon enteroscopy but missed by capsule endoscopy. Gastrointest Endosc 2006; 64: 445 - 449

[27] Clarke JO, Giday SA, Magno P et al. How good is capsule endoscopy for detection of periampullary lesions? Results of a tertiary-referral center. Gastrointest Endosc 2008; 68: 267-272

[28] Ou G, Shahidi N, Galorport C et al. Effect of longer battery life on small bowel capsule endoscopy. World J Gastroenterol 2015; 21: $2677-2682$ 